\title{
Paiche (Arapaima gigas) (Cuvier, 1829) (Osteoglossiformes: Arapaimidae): una revisión sistemática de la diversidad genética para la conservación del pez gigante del Amazonas
}

Paiche (Arapaima gigas) (Cuvier, 1829) (Osteoglossiformes: Arapaimidae): a systematic review of the genetic diversity for conservation of the amazon giant fish

Recibido: febrero 21 de 2021 | Revisado: marzo 02 de 2021 | Aceptado: abril 14 de 2021

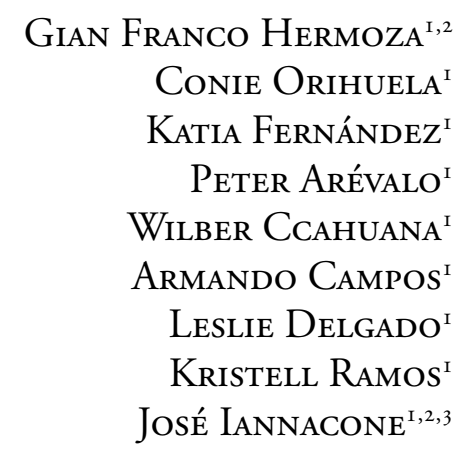

Grupo de Investigación en Sostenibilidad Ambiental (GISA), Escuela Universitaria de Posgrado (EUPG), Laboratorio de Ecología y Biodiversidad Animal (LEBA). Facultad de Ciencias Naturales y Matemática (FCCNM). Universidad Nacional Federico Villarreal (UNFV), Lima, Perú

2 Laboratorio de Ingeniería Ambiental. Carrera de Ingeniería Ambiental. Universidad Científica del Sur (UCSUR), Lima, Perú

3 Laboratorio de Parasitología. Facultad de Ciencias Biológicas. Universidad Ricardo Palma. Lima, Perú

Autor para correspondencia E-mail: jiannaconeo@unfv.edu.pe

Fuentes de financiamiento: Concurso de Investigación Formativa 2020 (Resolución R. No $7802-2020-C U-U N F V)$

\section{Resumen}

El paiche (Arapaima gigas) (Cuvier, 1829) (Osteoglossiformes: Arapaimidae) habita principalmente en los países de Perú, Brasil, Ecuador y Guyana. Se realizó un estudio de revisión sistemática exhaustivo sobre la diversidad genética para la conservación de $A$. gigas en la amazonía, usando como motores de búsqueda a Scielo, Google Académico, Google, Researchgate, Scopus, Reabic, PloS One, Springer, PubMed, BMC Genetics y Geneticsmr. com. Se utilizó el siguiente filtro de búsqueda: fecha de publicación: 2015 a febrero del 2021; tipo de publicaciones: artículos originales, notas científicas y libros; idioma: español, inglés y portugués; palabras claves de búsqueda en tres idiomas: "Aparaima gigas", "paiche", "conservación genética del paiche” y "genética de la conservación”. En las 14 publicaciones científicas recopiladas, se observa que la variabilidad genética se ve afectada por el distanciamiento de las poblaciones de $A$. gigas en las cuencas del Amazonas, y que diferentes poblaciones (metapoblaciones) de $A$. gigas se reproducen con poblaciones cercanas pudiendo generar posteriormente endogamia. Existe una mayor diferenciación genética entre las poblaciones de $A$. gigas a mayores distancias de separación. Un menor número de estudios enfatizaron el uso de los biomarcadores genéticos en $A$. gigas para una caracterización poblacional. Recientemente se están usando técnicas genético moleculares y de tecnologías con ADN ambiental en especies como el paiche. La insuficiencia de datos en esta especie para su manejo poblacional podría conllevar a una reducción de su diversidad genética o su extinción.

Palabras clave: Arapaima gigas, conservación, Sudamérica, variabilidad genética

\section{Abstract \\ The paiche (Arapaima gigas) (Cuvier, 1829) (Osteoglossiformes: Arapaimidae) lives mainly in the countries of Peru, Brazil, Ecuador and Guyana. An}

(C) Los autores. Este artículo es publicado por la Revista Campus de la Facultad de Ingeniería y Arquitectura de la Universidad de San Martín de Porres. Este artículo se distribuye en los términos de la Licencia Creative Commons Atribución No-comercial - Compartir-Igual 4.0 Internacional (https://creativecommons.org/licenses/ CC-BY), que permite el uso no comercial, distribución y reproducción en cualquier medio siempre que la obra original sea debidamente citada. Para uso comercial contactara: revistacampus@usmp.pe. 
exhaustive review study was carried out on genetic diversity for the conservation of $A$. gigas in the Amazon, using as search engines Scielo, academic Google, Google, Researchgate, Scopus, Reabic, PloS One, Springer, PubMed, BMC Genetics and Geneticsmr.com. The following search filter was used: publication date: 2015 to February 2021; type of publications: original articles, scientific notes and books; language: Spanish, English and Portuguese; Search keywords in three languages: "Aparaima gigas", "paiche", "genetic conservation of the paiche" and "genetics of conservation". In the 14 scientific publications compiled, it is observed that genetic variability is affected by the distancing of $A$. gigas populations in the Amazon basins, and different populations (metapopulations) of $A$. gigas reproduce with nearby populations, being able to subsequently generate inbreeding. There is greater genetic differentiation between populations of A. gigas at greater distances of separation. A smaller number of studies emphasized the use of genetic biomarkers in $A$. gigas for population characterization. Recently, molecular genetic techniques and technologies with environmental DNA are being used in species such as the paiche. Insufficient data on this species for population management could lead to a reduction in its genetic diversity or its extinction.

Key words: Arapaima gigas, conservation, South America, genetic variability

\section{Introducción}

Arapaima gigas (Schinz, 1822) habita en la cuenca amazónica, y es comúnmente llamado "paiche" en Perú y Ecuador, y "pirarucú” en Brasil (GarcíaDávila et al., 2018). Es considerado el pez dulceacuícola más grande del mundo, en la etapa adulta alcanzan hasta $250 \mathrm{~kg}$ y miden más de $3 \mathrm{~m}$ de longitud total (IIAP, 2017; Torres-Vásquez et al., 2017). La distribución geográfica natural de $A$. gigas incluye las cuencas de los ríos Amazonas, Tocantins-Araguaia y Esequibo que cubren Brasil, Ecuador, Guyana y Perú (Torati et al., 2019). También se han evaluado los principales problemas sanitarios en $A$. gigas (Murrieta et al., 2020) y su importancia en la cadena económica de valor (PNIPA, 2020).

Arapaima gigas fue listado en el Apéndice II que incluyen especies que no están necesariamente amenazadas de extinción pero que podrían llegar a estarlo a menos que se controle estrictamente su comercio, según la Convención sobre el Comercio Internacional de Especies Amenazadas de Fauna y Flora Silvestres (CITES) (Torati et al., 2019). Arapaima gigas presenta una disminución en sus poblaciones debido a la captura ilegal y a la pérdida del hábitat, y es todo un desafío para su conservación, por ello se debe realizar un manejo sostenible de esta especie (Hrbek et al., 2007; García-Dávila \& Renno, 2016; Hurd et al., 2016; IIAP, 2017). Existen registros que señalan a $A$. gigas como una especie exótica invasora cuando se le encuentra fuera de su ámbito de distribución natural. La presencia del paiche en la Reserva Nacional de Vida Silvestre Amazónica Manuripi (RNVSAM) en Bolivia, es un ejemplo de esta especie como invasora con un impacto negativo 
sobre la ictiofauna nativa de la reserva (Van Damme et al., 2011; Doria et al., 2020).

Por otro lado, en el Perú se presentan periodos de veda como una medida para la conservación de $A$. gigas (PRODUCE, 2020). Actualmente, esta especie se encuentra identificada como "Datos insuficientes" en la UICN (Unión Internacional para la Conservación de la Naturaleza) (Vitorino et al., 2017), lo que significa que hay un conocimiento insuficiente de su biología y genética para gestionar eficazmente su conservación (IIAP, 2017; Pires et al., 2019).

En la genética de la conservación, las técnicas moleculares proporcionan un dominio para entender la variación genética en poblaciones naturales de diferentes especies animales que van asociados a planes de manejo (Haig, 1998; Deyoung \& Honeycutt, 2005; Arif \& Khan, 2009; Morin et al., 2010; Allendorf, 2017; Coates et al., 2018; Holderegger $e t$ al., 2019). La diversidad genética de una población animal se considera la materia prima para la evolución por selección natural. Sin embargo, el acervo genético de una población animal puede cambiar con el tiempo, afectando el tamańo de la población y variando las tasas de natalidad, mortalidad y migración, los cuales están estrechamente relacionados con la variabilidad genética (Vitorino et al., 2015).

Por ende, uno de los principales problemas para el manejo y conservación de $A$. gigas radica en la poca información sistematizada sobre su estructura genética poblacional, los niveles de diversidad genética y el flujo de genes entre subpoblaciones, lo que conlleva a que los pobladores que consumen el paiche, piensen que se trata de una sola entidad genética, lo que podría ocasionar en una sobreexplotación de sus poblaciones, lo cual finalmente podría conducir a una disminución de su diversidad genética o a su extinción (Watson et al., 2016).

La genómica funcional se aplica cada vez más a las especies animales y emplean los métodos de la transcriptómica para mejorar el campo de la fisiología de la conservación de la biota animal (Connon et al., 2018). Estudios recientes han comenzado a reconocer algunas de estas posibilidades, cómo utilizar datos de la transcriptómica funcional para mejorar o perfeccionar un plan de manejo para la conservación de las especies animales (Coker, 2017; Connon et al., 2018); la tecnología de secuenciación molecular se puede aplicar a peces de alto interés usando las pruebas genéticas adecuadas; sin embargo, la sensibilidad de algunas especies protegidas o los resultados de estar incluidas en alguna lista de protección, hace que no puedan evaluarse debido a la escasa información sobre los parámetros claves en su ciclo de vida (Den Nijs, 2003; Connon et al., 2018).

Watson et al. (2016) mencionan que existen estudios de diferenciación genética en donde se han hecho uso de marcadores moleculares tipo microsatélites, lo que podría servir para determinar la genética de las poblaciones de $A$. gigas que habitan en diferentes cuencas del Amazonas. Por otro lado, la diferencia relativa genética entre las especies o dentro de cada especie ayuda a una identificación a nivel poblacional, por lo cual la detección del ADN sirve como una herramienta de detección sensible y precisa y, por lo tanto, útil para la investigación y para la 
protección de sus poblaciones (Den Nijs, 2003; Barnes \& Turner, 2016). Estos avances en instrumentos genómicos y en pruebas que usan métodos de muestreo no invasivos, a menudo son indispensables para las especies en riesgo ecológico, lo que ha ampliado enormemente el alcance y el valor del campo de investigación en genética de la conservación (Russello et al., 2020).

En el paiche, los estudios publicados sobre genética de la conservación hasta hace cinco años atrás han sido muy escasos, principalmente, los realizados por Brasil (Hrbek et al., 2005; Hamoy et al., 2008; Hrbek \& Fairas, 2008; Rosa et al., 2009; Araripe et al., 2013; Barrose, 2014) por Perú (García-Dávila et al., 2011), y uno de filogenia molecular de la familia Osteoglossidae por China $(\mathrm{Mu}$ et al., 2012). Por lo que es necesario evaluar qué se ha trabajado con esta especie sobre genética de la conservación principalmente en los últimos cinco años. Por lo tanto, el objetivo de esta investigación fue realizar un estudio de revisión sistemática exhaustiva sobre la diversidad genética para la conservación de $A$. gigas, y así contribuir a conservación de esta especie íctica.

\section{Método}

Se realizó una búsqueda bibliográfica exhaustiva y se empleó como motores de búsqueda a SciELO, Google Académico, Google, ResearchGate, Reabic, Plos one, Springer, PubMed, BMC Genetics y Geneticsmr.com. Se utilizó como filtro de búsqueda: fecha de publicación: 2015 a febrero 2021. Se empleó este periodo según los señalado por Martín \& Lafuente (2017), quienes indican que debe evaluarse la calidad de la referencias bibliográficas empleadas en base a su actualidad y pertinencia, al ser principalmente de los últimos cinco años; tipo de publicación: artículos originales, notas científica y libros; idioma: español, inglés y portugués; palabras claves de búsqueda: "Aparaima gigas", "paiche", "conservación genética del paiche", y "genética de la conservación" en los tres idiomas. Se empleó en todos los casos el operador boleano de búsqueda: "AND/Y" que recuperó documentos que contuvieran en pares, dos de los cuatro términos de búsqueda simultáneamente. Solo se incluyeron publicaciones científicas que brindaron información sobre el uso de la genética para conservación de $A$. gigas. Se excluyeron las publicaciones que no brindaban información de acorde a los objetivos de la búsqueda. Se recuperó un total de 28 publicaciones de las cuales se eliminaron dos por estar duplicadas, y se excluyeron 11 por no cumplir los criterios anteriormente mencionados. Finalmente se trabajó con 14 publicaciones científicas, 12 artículos originales, una nota científica y un libro (Figura 1).

La información fue, finalmente, organizada por autor, año, país, lugar de estudio, población estudiada, buscador seleccionado y algún detalle importante de cada publicación. 


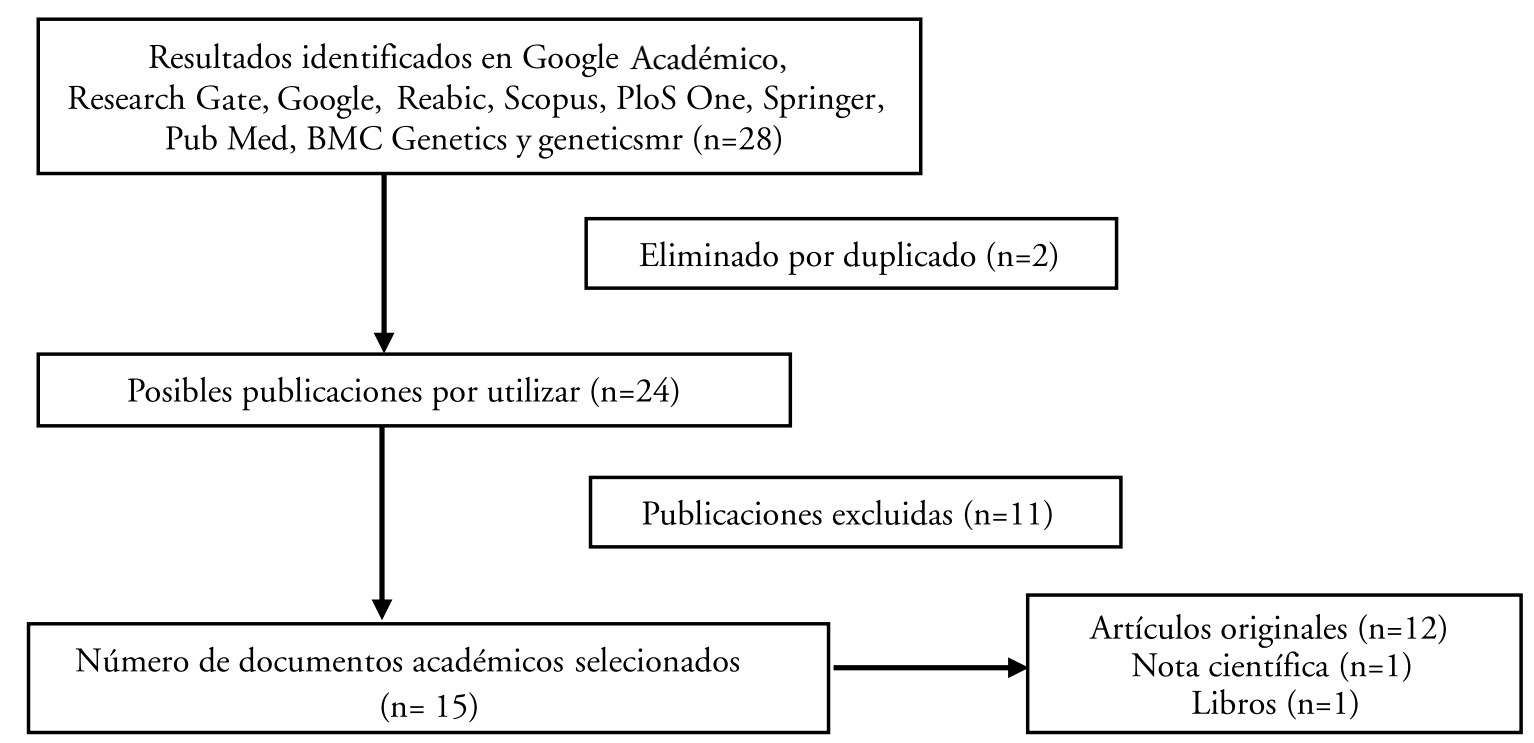

Figura 1. Flujograma de la búsqueda bibliográfica sobre genética para conservación en Arapaima gigas.

Aspectos éticos: Los autores declaran que se cumplieron todos los aspectos éticos nacionales e internacionales para efectuar la siguiente revisión.

\section{Resultados y Discusión}

El análisis con relación al año de estudio mostró que en el 2015 se observó una sola en el 2016, tres, en el 2017 cuatro, en el 2018 una, en el 2019 tres, en el 2020 una y finalmente hasta febrero del 2021, una publicación, respectivamente. De las 14 publicaciones académicas consideradas para el desarrollo del presente trabajo, cinco no especificaron la población estudiada, y tres no especificaron ni la población estudiada ni el lugar de estudio respectivamente (Tabla 1 ). También, la mayor cantidad de literatura científica fue de Brasil ( $n=7)$ seguida de Estados Unidos $(\mathrm{n}=4)$, Perú $(\mathrm{n}=1)$, Bolivia $(\mathrm{n}=1)$, Alemania $(n=1)$ y China $(n=1)$ (Tabla 1). De los 14 documentos científicos, 12 fueron en el idioma inglés $(85,71 \%)$, y dos en espańol (14,29\%). El buscador Google académico recuperó el mayor número de documentos científicos, seguido por PubMed (Tabla 1).

De los 14 trabajos seleccionados, la temática de variabilidad genética en $A$. gigas para la evaluación de poblaciones en forma comparativa fue evaluada en al menos siete documentos científicos (Vitorino et al., 2015; García-Dávila \& Renno, 2016; Allendorf, 2017; FazziGomes et al., 2017; Pires et al., 2019; Torati et al., 2019; Nogueira et al., 2020). Así, se hizo una comparación entre diversas poblaciones de $A$. gigas estudiadas en Brasil, y se observó una mayor diversidad genética en la población de nuevo San Antonio con 2,43 de variabilidad genética (Vitorino et al., 2017), seguida de Karanambu con 2,02, e Iwokrama con 1,53 y finalmente Araguaiana con una variabilidad genética de 1,52 (Watson et al., 2016; Vitorino et al., 2017), comprobándose lo mencionado por García-Dávila \& Renno (2016), quienes indican que las poblaciones alejadas o que se encuentran en diferentes cuencas pueden presentar 
una mayor variabilidad genética. Esto es producido porque $A$. gigas se encuentra disperso en diferentes poblaciones ("metapoblaciones") reproduciéndose solo con poblaciones cercanas, lo que puede aumentar la endogamia (FazziGómez et al., 2017). Por lo tanto, cruzar individuos genéticamente distintos puede tener efectos en su variabilidad genética (Watson et al., 2016), debido a que no se adapta a un medio ocupado por una población diferente de una misma especie en base a la resistencia o susceptibilidad en su hábitat. Se recomienda utilizar en la reproducción a individuos de poblaciones cercanas, pero vigilando el no favorecer la endogamia (García-Dávila \& Renno, 2016). Las variabilidades genéticas mencionadas anteriormente podrían estar relacionadas con características adaptativas, por ejemplo, una mayor producción de secreciones de la glándula de la cabeza, silenciamiento o sobreexpresión del gen relacionado a la talla y el crecimiento acelerado (Du et al., 2019). GarcíaDávila \& Renno (2016) indican que las poblaciones amazónicas peruanas de $A$. gigas tienen un flujo genético reducido debido a las distancias geográficas entre sus poblaciones.

Otros estudios son en el campo de la citogenética para promover el desarrollo de las pesquerías en la Amazonía, y mencionan el cariotipo de 28 cromosomas en el paiche, que no permite una adecuada diferenciación el sexo (Carvajal-Vallejos et al., 2017); contrario a lo que menciona Du et al. (2019) que indican que se puede reconocer el sexo de $A$. gigas mediante el análisis de cromosomas. También se puede analizar etiquetas RAD (sitios de restricción) (García-Dávila \& Renno, 2016), las cuales están presentes en la mayoría de los machos, pero ausentes en las hembras, de igual manera existen equipos que pueden identificar el género mediante la hormona vitelogénica (García-Dávila $\&$ Renno, 2016; Fernandino \& Hattori, 2019).

Nogueira et al. (2020) compararon poblaciones de $A$. gigas entre sitios de muestreo de la Cuenca del Amazonas y de la Cuenca Tocantins-Araguaia, Brasil, revelando altos niveles de variabilidad genética en la cuenca del Amazonas, con diversidad de haplotipos, y diversidad de nucleótidos con respecto a los sitios en la cuenca Tocantins-Araguaia, los cuales exhibieron niveles moderados a bajos de variabilidad genética, con diversidad de haplotipos que van de cero a 0,6 y con diversidad de nucleótidos de cero. Estos resultados fueron expuestos con anterioridad por Pires et al. (2019), los cuales evidenciaron la fragilidad de las poblaciones de $A$. gigas en las cuencas brasileñas (Araguaia-Tocantins y Amazonas), y la consideraron una especie vulnerable en temas de reducción de población a nivel del río Araguaia por la pobreza en variabilidad o capacidad genética reducida en la estructuración entre poblaciones de cuatro localidades de los ríos Araguaia y Tocantins. A su vez esto fue confirmado con el análisis de las muestras de la población de la cuenca del Amazonas (Amazonas y Solimóes), las cuales fueron genéticamente más diversas que las de los ríos Araguaia y Tocantins en términos de porcentaje de loci polimórficos, número de alelos privados, índice de Shannon-Weaver, índice de información (I) y de heterocigosidad observada (HO). De hecho, un estudio que evaluó la capacidad de dispersión de A. gigas, concluyó la existencia de altos 
niveles de similitud genética entre lagos separados por $25 \mathrm{~km}$, diferenciación genética moderada en sitios separados por $100 \mathrm{~km}$ y mayor diferenciación genética entre regiones separadas mayores a 1500 $\mathrm{km}$, confirmado con observaciones previas (Torati et al., 2019).

Cinco estudios enfatizaron el uso de los biomarcadores genéticos en $A$. gigas para una caracterización genética (Watson $e t$ al., 2016; Vitorino et al., 2017; Torati et al., 2019; Fazzi-Gomes et al., 2021). Diversas técnicas genético moleculares y de tecnologías con ADN ambiental están usándose en especies de interés, entre ellas el paiche para la investigación de este recurso hidrobiológico y para su conservación (Barnes \& Turner, 2016; Connon et al., 2018). La insuficiencia de datos de esta especie para su manejo poblacional podría conllevar a una reducción de su diversidad genética o su extinción.

Tabla 1

Publicaciones consideradas según el contenido de interés de la revisión sistemática de la variabilidad genética del paiche (Arapaima gigas).

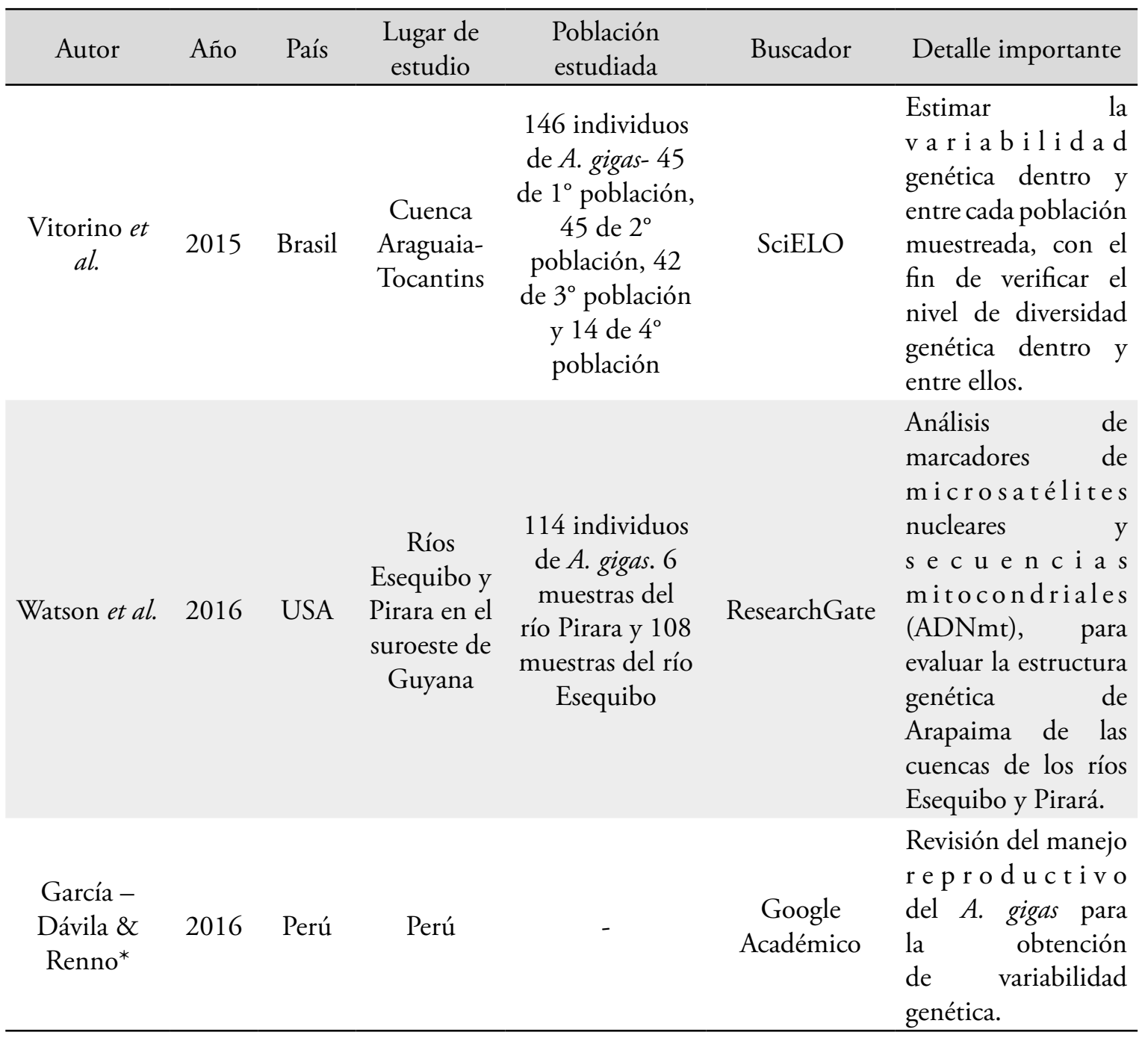


Gian Franco Hermoza - Conie Orihuela - Katia Fernández - Peter Arévalo - Wilber Ccahuana Armando Campos - Leslie Delgado - Kristell Ramos - José Iannacone

Tabla 2

Publicaciones consideradas según el contenido de interés de la revisión sistemática de la variabilidad genética del paiche (Arapaima gigas) (continuación).

\begin{tabular}{|c|c|c|c|c|c|c|}
\hline Autor & Año & País & $\begin{array}{l}\text { Lugar de } \\
\text { estudio }\end{array}$ & $\begin{array}{l}\text { Población } \\
\text { estudiada }\end{array}$ & Buscador & Detalle importante \\
\hline $\begin{array}{l}\text { Barnes \& } \\
\text { Turner }\end{array}$ & 2016 & USA & - & - & Springer & $\begin{array}{l}\text { La aplicación de } \\
\text { tecnología basada en ADN } \\
\text { ambiental (ADNe) como } \\
\text { herramienta emergente } \\
\text { para la investigación y la } \\
\text { conservación. }\end{array}$ \\
\hline $\begin{array}{l}\text { Carvajal- } \\
\text { Vallejos et } \\
\text { al. }{ }^{* *}\end{array}$ & 2017 & Bolivia & $\begin{array}{l}\text { Cuenca } \\
\text { Amazónica } \\
\text { Boliviana }\end{array}$ & $\begin{array}{c}802 \\
\text { especímenes } \\
\text { de } A \text {. gigas } \\
\text { validas, } 12 \\
\text { de ellas no } \\
\text { nativas }\end{array}$ & $\begin{array}{c}\text { Google } \\
\text { Académico }\end{array}$ & $\begin{array}{l}\text { Estrategias para promover } \\
\text { el desarrollo de las } \\
\text { pesquerías amazónicas. }\end{array}$ \\
\hline $\begin{array}{l}\mathrm{F} \text { a z z i - } \\
\text { Gomes et } \\
\text { al. }\end{array}$ & 2017 & Brasil & $\begin{array}{l}\text { Lago Grande } \\
\text { Curuai, lago } \\
\text { Paru (Pará) }\end{array}$ & $\begin{array}{l}87 \text { muestras } \\
\text { de } A \text {. } \\
\text { gigas ( } 2 \\
\text { poblaciones) }\end{array}$ & Geneticsmr & $\begin{array}{l}\text { Análisis de la diversidad } \\
\text { genética y la diferenciación } \\
\text { poblacional de dos } \\
\text { poblaciones naturales } \\
\text { de } A \text {. gigas. Se usaron } \\
\text { biomarcadores } \\
\text { microsatélites }\end{array}$ \\
\hline $\begin{array}{l}\text { Vitorino et } \\
\text { al. }\end{array}$ & 2017 & Brasil & $\begin{array}{l}\text { Cuenca del } \\
\text { Araguaia- } \\
\text { Tocantins }\end{array}$ & $\begin{array}{c}294 \\
\text { muestras } \\
\text { de tejido } \\
\text { muscular } \\
\text { y aletas de } \\
\text { A. gigas } \\
\text { de cinco } \\
\text { lugares } \\
\text { diferentes }\end{array}$ & SciELO & $\begin{array}{l}\text { Análisis de biomarcadores } \\
\text { microsatélites para evaluar } \\
\text { la diversidad genética y } \\
\text { la conectividad de cinco } \\
\text { poblaciones silvestres de } A \text {. } \\
\text { gigas. }\end{array}$ \\
\hline Allendorf & 2017 & USA & - & - & PubMed & $\begin{array}{l}\text { Estudio de la variación } \\
\text { genética para conservar } \\
\text { las poblaciones naturales } \\
\text { desde los últimos } 50 \text { ańos }\end{array}$ \\
\hline $\begin{array}{l}\text { Connon et } \\
\text { al. }\end{array}$ & 2018 & USA & - & - & PubMed & $\begin{array}{l}\text { Aplicabilidad de los } \\
\text { métodos a especies de } \\
\text { interés. }\end{array}$ \\
\hline
\end{tabular}


Paiche (ARapaima gigas) (Cuvier, i 829) (Osteoglossiformes: Arapaimidae): una Revisión Sistemática de la DIVERSIDAD GENÉTICA PARA LA CONSERVACIÓN DEL PEZ GIGANTE DEL AMAZONAS

Tabla 3

Publicaciones consideradas según el contenido de interés de la revisión sistemática de la variabilidad genética del paiche (Arapaima gigas) (continuación)

\begin{tabular}{|c|c|c|c|c|c|c|}
\hline Autor & Año & País & $\begin{array}{c}\text { Lugar de } \\
\text { estudio }\end{array}$ & $\begin{array}{l}\text { Población } \\
\text { estudiada }\end{array}$ & Buscador & $\begin{array}{c}\text { Detalle } \\
\text { importante }\end{array}$ \\
\hline $\begin{array}{c}\text { Pires et } \\
\text { al. }\end{array}$ & 2019 & Brasil & $\begin{array}{l}19 \text { localidades } \\
\text { de la cuenca del } \\
\text { Amazonas y } \\
\text { tres de la cuenca } \\
\text { de Tocantins- } \\
\text { Araguaia }\end{array}$ & $\begin{array}{c}385 \text { individuos } \\
\text { de } \text { A. gigas de } \\
\text { las Cuencas del } \\
\text { Amazonas y } 126 \\
\text { de la Cuenca de } \\
\text { Tocatins-Araguaia }\end{array}$ & PloS One & $\begin{array}{l}\text { Distribución de } \\
\text { la diversidad } \\
\text { genética y el } \\
\text { patrón de la } \\
\text { estructura de la } \\
\text { población de } A \text {. } \\
\text { gigas en diferentes } \\
\text { localidades. }\end{array}$ \\
\hline $\begin{array}{c}\text { Torati et } \\
\text { al. }\end{array}$ & 2019 & Brasil & $\begin{array}{l}\text { Ríos Amazonas, } \\
\text { Solimões, } \\
\text { Tocantins, } \\
\text { Araguaia y } \\
\text { una población } \\
\text { cautiva }\end{array}$ & $\begin{array}{l}60 \text { individuos de } \\
\text { A. gigas, } 12 \text { por } \\
\text { cada población }\end{array}$ & $\begin{array}{l}\text { BMC } \\
\text { Genetics. } \\
\text { Scopus }\end{array}$ & $\begin{array}{l}\text { Evaluación de } \\
\text { la diversidad y } \\
\text { estructura génica } \\
\text { a través de la } \\
\text { secuenciación de } \\
\text { Polimorfismos } \\
\text { Genómicos. }\end{array}$ \\
\hline Du et al. & 2019 & $\begin{array}{l}\text { Brasil, } \\
\text { China, } \\
\text { Alemania }\end{array}$ & Brasil & - & $\begin{array}{l}\text { Scopus, } \\
\text { PubMed }\end{array}$ & $\begin{array}{l}\text { Secuenciamiento } \\
\text { genético gigas, } \\
\text { A. de o m a ción } \\
\text { co m a r ét ic a; } \\
\text { filog en estudio de genes } \\
\text { en importancia } \\
\text { morfológica y } \\
\text { reproductiva. }\end{array}$ \\
\hline $\begin{array}{l}\text { Nogueira } \\
\text { et al. }\end{array}$ & 2020 & Brasil & $\begin{array}{c}\text { Cuenca del } \\
\text { Amazonas } \\
\text { y cuenca de } \\
\text { Tocantins- } \\
\text { Araguaia }\end{array}$ & $\begin{array}{c}121 \text { muestras } A \text {. } \\
\text { gigas de cinco } \\
\text { sitios de la cuenca } \\
\text { del Amazonas } \\
\text { y } 111 \text { muestras } \\
\text { de cinco sitios } \\
\text { de la cuenca } \\
\text { de Tocantins- } \\
\text { Araguaia }\end{array}$ & $\begin{array}{l}\text { Google } \\
\text { Académico, } \\
\text { PubMed }\end{array}$ & $\begin{array}{l}\text { Análisis genético } \\
\text { del arapaima } \\
\text { utilizando PCR y } \\
\text { secuenciamiento } \\
\text { genómico a partir } \\
\text { de DNA genómico } \\
\text { para caracterizar } \\
\text { la variación } \\
\text { genética entre } \\
\text { estas cuencas. }\end{array}$ \\
\hline $\begin{array}{l}\text { Fazzi- } \\
\text { Gomes et } \\
\text { al. }\end{array}$ & 2021 & Brasil & $\begin{array}{l}\text { "Figshare online } \\
\text { data" } \\
\text { repositorio }\end{array}$ & $\begin{array}{c}\text { Genoma completo } \\
\text { de } A \text {. gigas }\end{array}$ & $\begin{array}{c}\text { Google } \\
\text { Académico }\end{array}$ & $\begin{array}{l}\text { Los marcadores } \\
\text { de microsatélites. } \\
\text { Herramientas son } \\
\text { valiosas para la } \\
\text { investigación en } \\
\text { bioinformática, } \\
\text { ecología, genética, } \\
\text { e v o l u c i ó n } \\
\text { y estudios } \\
\text { comparativos. }\end{array}$ \\
\hline
\end{tabular}

${ }^{*}=$ Nota científica. ${ }^{* *}=$ Libro. 


\section{Conclusiones}

La revisión sistemática de la literatura encontró 14 publicaciones científicas que documentan la diversidad genética, y métodos genéticos y moleculares aplicables para el estudio de la variabilidad genética para su conservación en las diferentes poblaciones de $A$. gigas. La mayor cantidad de literatura científica fue de Brasil seguida de Estados Unidos. El buscador Google Académico recuperó el mayor número de documentos, seguido por PubMed. 12 documentos científicos fueron en el idioma inglés $(85,71 \%)$, y dos en español $(14,29 \%)$.
La temática de variabilidad genética en A. gigas para la evaluación de poblaciones en forma comparativa fue evaluada en al menos siete documentos científicos. Las metapoblaciones de $A$. gigas que pueblan en la cuenca amazónica poseen una variabilidad genética entre sus poblaciones, por lo que es idóneo un cruzamiento entre poblaciones cercanas para su conservación. Un menor número de estudios enfatizaron el uso de los biomarcadores genéticos en $A$. gigas para una caracterización poblacional. Recientemente se están usando técnicas genético moleculares y de tecnologías con ADN ambiental en $A$. gigas.

\section{Referencias}

Allendorf, F. (2017). Genetics and the conservation of natural populations: allozymes to genomes. Molecular Ecology, 26, 420-430.

Araripe, J., Rêgo, P.S.; Queiroz, H., Sampaio, I. \& Schneider, H.(2013). Dispersal capacity and genetic structure of Arapaima gigas on different geographic scales using microsatellite markers. PLoS ONE, 8: e54470.

Arif, I.A. \& Khan, H.A. (2009). Molecular markers for biodiversity analysis of wildlife animals: a brief review. Animal Biodiversity and Conservation, 32, 9-17.

Barnes, A. \& Turner, R. (2016). The ecology of environmental DNA and implications for conservation genetics. Conservation Genetics, 1, $1-17$.

Barrose, C.V. (2014). Genetic associations amongstsubpopulation of Arapaima gigas. Advances in Aquaculture and Fisheries Management, 2, 206-216.

Carvajal-Vallejos, F., Salas, R., Navia, J., Carolsfeld, J. \& Van Damme, P.(2017). Bases técnicas para el manejo y aprovechamiento del paiche (Arapaima gigas) en la cuenca amazónica boliviana. Instituto Nacional de Innovación Agropecuaria y Forestal. Bolivia.

Coates, D.J., Byrne, M. \& Moritz, C. (2018). Genetic diversity and conservation units: dealing with the species-population continuum in the age of genomics. Frontiers in Ecology and Evolution, 6: 165.

Coker,O.M. (2017). Importance of genetics in conservation of biodiversity. Nigerian Journal of Wildlife Management, 1 (Special Edition): 11-18.

Connon, R., Jeffries, K., Komoroske, L., Todgham, A. \& Fangue, 
N. (2018). The utility of transcriptomics in fish conservation. Journal of Experimental Biology, 221, jeb148833.

Den Nijs, H.C.M. (2003). Conservation and molecular methods. In: Ammann, K., Jacot, Y. \& Braun, R. (eds). Methods for Risk Assessment of Transgenic Plants. Birkhäuser, B. https://doi.org/10.1007/978-30348-8033-6_13

Deyoung, R.W.\& Honeycutt, R.L. (2005). The molecular toolbox: genetic techniques in wildlife ecology and management. Journal of Wildlife Management, 69, 1362-1384.

Doria, C.R.C., Catâneo, D.T.B.S. Torrente-Vilara, G. \& Vitule, J.R.S. (2020). Is there a future for artisanal fishing in the Amazon? The case of Arapaima gigas. Management of Biological Invasions, 11, 1-8.

Du, K., Wuertz, S., Adolfi, M., Kneitz, S., Stöck, M., Oliveira, M., Nóbrega, R., Ormanns, J., Kloas, W., Feron, R., Klopp, C., Parrinello, H., Journot, L., He, S., Postlethwait, J., Meyer, A., Guiguen, Y. \& Schartl, M. (2019). The genome of the arapaima (Arapaima gigas) provides insights into gigantism, fast growth and chromosomal sex determination system. Scientific reports, 9, 1-11.

Farias, I.P., Hrbek, T., Brinkmann, H., Sampaio, I. \& Meyer, A. (2003). Characterization and isolation of DNA microsatellite primers for Arapaima gigas, an economically important but severely over- exploited fish species of the Amazon basin. Molecular Ecology Notes, 3, 128-130.

Fazzi-Gomes, P., Melo, N., Palheta, G., Guerreiro, S., Amador, M., Ribeirodos-Santos, K. \& Hamoy, I. (2017). Rapid-Communication Genetic diversity and differentiation in natural populations of Arapaima gigas from lower Amazon revealed by microsatellites. Genetics and Molecular Research, 16, gmr16019552.

Fazzi-Gomes, P., Aguiar, J., Cabral, G.F., Marques, D., Palheta, H., Moreira, F., Rodrigues, M., Cavalcante, R., Souza, J., Silva, C., Hamoy, I. \& Santos, S. (2021). Genomic approach for conservation and the sustainable management of endangered species of the Amazon. PLoS ONE, 16: e0240002.

Fernandino, J.I. \& Hattori, R.S. (2019). Sex determination in Neotropical fish: Implications ranging from aquaculture technology to ecological assessment. General and Comparative Endocrinology, 273, 172-183.

García-Dávila, R. \& Renno, F. (2016). Manejo genético de reproductores de paiche Arapaima gigas para reproducción en cautiverio. Folia Amazónica, 25, 179-182.

García-Dávila, C., Castro-Ruiz, D., Chota-Macuyama, W., Biff, C., Deza, S., Bazan, R., Garcia, J., Rebaza, M., Rebaza, C., Chavez, C., Chu-Koo, F., Duponchelle, F., Nuñez, J. \& Renno, J.F. 
(20119. Caracterización genética de ejemplares de paiche Arapaima gigas (Cuvier, 1829) utilizados en el repoblamiento del lago Imiria (cuenca del río Ucayali). Folia amazónica, 20, 67-75.

García-Dávila, C., Sánchez, H., Flores, M., Mejía, J., Angulo, C., CastroRuiz, D., Estivals, G., García, A., Vargas, G., Nolorbe, C., Núñez, J., Mariac, C., Duponchelle, F. \& Renno, J.F. (2018). Peces de consumo de la Amazonía peruana. Instituto de Investigaciones de la Amazonía Peruana (IIAP). Iquitos, Perú. 218 pp.

Haig, S.M. (1998). Molecular contributions to conservation. Ecology, 79, 413-425.

Hamoy, I.G., Santos, E.J.M. \& Santos, S.E.B. (2008). Rapid and inexpensive analysis of genetic variability in Arapaima gigas by PCR multiplex panel of eight microsatellites. Genetics and Molecular Research, 7, 29-32.

Henrique, C., Leitão, C.S.S., Paula e Silva, M.N. \& Almeida e Val, M.F. (2014). Genetic relationships between captive and wild subpopulations of Arapaima gigas (Schinz, in Cuvier, 1822). International Journal of Fisheries and Aquaculture, 6, 108-123.

Holderegger, R., Balkenhol, N., Bolliger, J., Engler, J.O., Gugerli, F., Hockkirch, A., Carten, N.,Segelbacher, G., Widmer, A. \& Zachos, F.E. (2019). Conservation genetics: Linking science with practice. Molecular Ecology, 28: 3848-3856.

Hrbek, T. \& Fairas, I. (2008). The complete mitochondrial genome of the pirarucu (Arapaima gigas, Arapaimidae, Osteoglossiformes). Genetics and Molecular Biology, 31, 293-302.

Hrbek, T., Farias, I.P., Crossa, M., Sampaio, I., Porto, J.I.R. \& Meyer, A. (2005). Population genetic analysis of Arapaima gigas, one of the largest freshwater fishes of the Amazon basin: implications for its conservation. Animal Conservation, 8, 297-308.

Hrbek, T., Crossa, M. \& Farias, I.P. (2007). Conservation strategies for Arapaima gigas (Schinz, 1822) and the Amazonian várzea ecosystem. Brazilian Journal of Biology, 67(4, Suppl.), 909-917.

Hurd, L.E., Sous, R.G.C., Siqueira-Souza, F.K., Cooper, G.J., Kahne, J.R. \& Freitas, C.E.C. (2016). Amazon floodplain fish communities: Habitat connectivity and conservation in a rapidly deteriorating environment. Biological Conservation, 195, 118127.

Instituto de Investigaciones de la Amazonía Peruana (IIAP). (2017). El cultivo del paiche. Biología, procesos productivos, tecnologias $y$ estadísticas. Primera Edición. IIAP. Ministerio del Ambiente/ Gobierno del Perú. Iquitos.

Martín, S.G. \& Lafuente, V. (2017). Referencias bibliográficas: 
indicadores para su evaluación en trabajos científicos. Investigación bibliotecológica, 31, 151-180.

Morin, P.A., Martien, K.K., Archer, F.I., Cipriano, F., Steel, D., Jackson, J. \& Taylor, B.L. (2010). Applied conservation genetics and the need for quality control and reporting of genetic data used in fisheries and wildlife management. Journal of Heredity, 101, 1-10.

Mu, X.D., Gu, D., Yang, Y., Luo, D., Meng, X., Wang, X., Hu, Y.C., \& Lu, J. (2013). Genetic diversity and phylogeny of the family Osteoglossidae by the nuclear $18 \mathrm{~S}$ ribosomal RNA and implications for its conservation. Biochemical Systematics and Ecology, 51, 280287.

Murrieta, G., Pereira, J. N. \& Yunis, J. (2020). Principales problemas sanitarios y enfermedades parasitarias en la crianza del paiche Arapaima gigas en la Amazonía. Instituto de Investigaciones de la Amazonía Peruana (IIAP). Iquitos-Perú. 57 pp.

Nogueira, F., Rêgo, Péricles, S., Queiroz, H., Venere, P., Varela, E., Sampaio, I., Schneider, H. \& Araripe, J. (2020). Genetic diversity and structuring in the Arapaima (Osteoglossiformes, Osteoglossidae) population reveal differences between the Amazon and the Tocantins-Araguaia basins. Anais da Academia Brasileira de Ciências, 92, e20180496.

Pires, I., Willis, S., Leão, A., Verba, J.T., Crossa, M., Foresti, F., Porto-
Foresti, F., Sampaio, I. \& Hrbek, T. (2019). The largest fish in the world's biggest river: Genetic connectivity and conservation of Arapaima gigas in the Amazon and Araguaia Tocantins drainages. PLoS One, 14, e0220882.

PNIPA (Programa Nacional de Innovación en Pesca y Acuicultura). (2020). Cadena de valor del Paiche. Hoja de ruta de la I\&D+i. Programa Nacional de Innovación en Pesca y Acuicultura - PNIPA. Lima.

PRODUCE (Ministerio de Producción). (2020). Resolución Ministerial No 00320-2020-PRODUCE. Perú. Recuperado el 2 de diciembre del 2020 de: https:// cdn.www.gob.pe/uploads/ document/file/1326281/R.\%20 M. \% $20 \mathrm{~N} \%$ C $2 \%$ B $0 \% 20$ $320-2020$ - P R O D U CE. pdf?fbclid=IwAR 1 fjP2PtgDCXhgY5bEF2V21fkZyfPkG2PZ2BBP 7UDhsQc41Ff3UgwIcw8

Rosa, R., Marceléia, R., Caetano-Filho, M. \& Giuliano-Caetano, L. (2009). Conserved Cytogenetic Features in the Amazonian Arapaima, Arapaima gigas (Schinz 1822) from Jamari River, Rondônia-Brazil. The Open Biology Journal, 2, 9194.

Russello, M., Waterhouse, M., Etter, P. \& Johnson, E. (2020).Conservation Genetics and Genomics. Genes, 11,318 .

Torati, L., Bernard, J., Sousa, E., Araripe, J., Wehne, S. \& Migaud, H. (2019). Genetic diversity 
and structure in Arapaima gigas populations from Amazon and Araguaia-Tocantins river basins. BMC Genetics, 20, 13.

Torres-Vásquez, M.I., Sampaya-Ruíz, J., Calderón-Robles, E. \& ArimuyaSalas, F. (2017). Monitoreo de la abundancia de Arapaima gigas en la cuenca baja del río Pacaya, Reserva Nacional Pacaya Samiria, Amazonía peruana. Folia Amazónica, 26, $175-186$.

Van Damme, P.A., Carvajal-Vallejos, F.M. \& Molina-Carpio, J. (Eds.). (2011). Los peces y delfines de la Amazonía boliviana: hábitats, potencialidades y amenazas. Edit. INIA, Cochabamba, Bolivia. 490 p.

Vitorino, C., Oliveira, R., Margarido. \& Venere, P. (2015). Genetic diversity of Arapaima gigas

(Schinz, 1822) (Osteoglossiformes:

Arapaimidae) in the Araguaia-

Tocantins basin estimated

by ISSR marker. Neotropical Ichthyology, 13, 557-568.

Vitorino, C.A., Nogueira, F., Souza, I.L., Araripe, J. \& Venere, P.C. (2017). Low genetic diversity and structuring of the Arapaima (Osteoglossiformes, Arapaimidae) population of the AraguaiaTocantins basin. Frontiers in Genetics, 8, 159.

Watson, L.C., Stewart, D.J. \& Kretzer, A.M. (2016). Genetic diversity and population structure of the threatened Giant Arapaima in southwestern Guyana: implications for their conservation. Copeia, 104, 864-872. 